\title{
PLETHORA proteins as dose-dependent master regulators of Arabidopsis root development
}

\author{
Carla Galinha ${ }^{1 \star} \uparrow$, Hugo Hofhuis ${ }^{1 \star}$, Marijn Luijten ${ }^{1}$, Viola Willemsen ${ }^{1}$, Ikram Blilou $^{1}$, Renze Heidstra ${ }^{1} \&$ Ben Scheres $^{1}$
}

Factors with a graded distribution can program fields of cells in a dose-dependent manner ${ }^{1,2}$, but no evidence has hitherto surfaced for such mechanisms in plants. In the Arabidopsis thaliana root, two PLETHORA (PLT) genes encoding AP2-domain transcription factors have been shown to maintain the activity of stem cells ${ }^{3}$. Here we show that a clade of four PLT homologues is necessary for root formation. Promoter activity and protein fusions of PLT homologues display gradient distributions with maxima in the stem cell area. PLT activities are largely additive and dosage dependent. High levels of PLT activity promote stem cell identity and maintenance; lower levels promote mitotic activity of stem cell daughters; and further reduction in levels is required for cell differentiation. Our findings indicate that PLT protein dosage is translated into distinct cellular responses.

During animal development, instructive molecules acquire a graded distribution and induce distinct cellular responses in a concentration-dependent manner. Whether similar mechanisms occur in plants has been controversial; dosage-sensitive action of plant hormones has been inferred only after external application ${ }^{4}$. Plant stem cell regions, which supply cells for the growing root and shoot systems ${ }^{5}$, are potential sites of action for instructive gradients. Stem cells are maintained in local micro-environments, which are similar to animal stem cell niches ${ }^{6}$. Stem cell daughters undergo additional divisions in transit-amplifying cell compartments called meristems; when cells leave the meristem they rapidly expand and differentiate. The PLETHORA1 (PLT1, At3g20840) and PLT2 (At1g51190) genes encode AP2-domain transcription factor family members essential for defining the root stem cell niche ${ }^{3}$. plt1;plt2 mutants display stem cell loss, loss of transit-amplifying cells and reduced cell expansion. PLT1 and PLT2 expression strongly correlates with a transcriptional response maximum to the plant hormone auxin in the root tip ${ }^{3,7}$ and this maximum has been shown to have profound organizing activity ${ }^{8}$ - a property often associated with sources of instructive gradients. Here, we reveal that the PLT gene family controls distinct aspects of root development in a dose-dependent manner through PLT expression gradients that culminate in the stem cell niche.

The proteins encoded by At5g10510/AINTEGUMENTA-LIKE6 (AIL6)/PLT3 and At5g17430/BABY BOOM(BBM) group with PLT1 and PLT2 in the AP2/ERF transcription factor family (Supplementary Fig. 1$)^{9}$, and these candidate redundant factors are predicted to be expressed in the $\operatorname{root}^{10}$.

From the heart-stage of embryogenesis onward, PLT3 is expressed in provascular cells, the quiescent centre and columella progenitor cells (Fig. 1a). Post-embryonically, PLT3 messenger RNA accumulates in the root stem cell niche with the strongest signal in the columella stem cell layer (Fig. 1b), in contrast to the predominant quiescent-centre-localization of PLT1 and PLT2 transcript ${ }^{3}$. At the heart-stage of embryo development, $B B M$ is expressed in provascular cells and in the lens-shaped quiescent centre progenitor cell (Fig. 1c). Post-embryonically, $B B M$ transcript accumulates in the quiescent centre and columella stem cells-in a similar manner to the PLT mRNAs - and in provascular cells of the proximal meristem (Fig. 1d).

The plt3-1 mutant allele carries a T-DNA insertion interrupting the first AP2 domain (Supplementary Fig. 2). No transcript was detected by PCR with reverse transcription (RT-PCR) or by in situ hybridization on plt3-1 seedlings (data not shown), suggesting that plt3-1 is a null allele. Homozygous plt3 single mutants have slightly shorter roots and meristems compared to wild type, but plt1 $1^{-/-}$ plt $2^{-1-}$ plt $3^{-1-}$ triple homozygotes are rootless (Fig. 1e, upper inset). Progeny from $p l t 1^{-/-}$plt $2^{-/-}$plt $3^{+/-}$, plt $1^{-/-}$plt $^{+/-}$plt $3^{-/-}$ and plt ${ }^{+/}$plt $2^{-/-}$plt $^{-/-}$plants segregate $\sim 25 \%$ rootless triple mutants (Supplementary Table 2), demonstrating linkage between the rootless phenotype and the three PLT genes. The embryonic root pole of triple homozygous seedlings is fully differentiated at 3 days post germination (d.p.g.) and adventitious root primordia arrest at 6 d.p.g. (Supplementary Fig. 3). Mature $p l t 1^{-1-} p l t 2^{-/-}$embryos have only subtle defects in the cellular organization of the distal-most region $^{3}$ (Supplementary Fig. 4), but plt $1^{+/-}$plt $^{-/-}$plt $3^{-/-}$parents yield $\sim 25 \%$ embryos with aberrant root poles that lack a lateral root cap cell layer (Supplementary Fig. 4).

We previously showed that plt1 $1^{-/-}$plt $2^{-/-}$mutants have strongly reduced transcription of the PIN4 gene, which encodes an auxin efflux facilitator ${ }^{11}$. In triple mutant embryos from $\mathrm{plt}^{+/-} \mathrm{plt}^{-/-}$ plt $3^{-/-}$parents, PIN1 and PIN3 mRNAs are strongly reduced (Fig. 1g-j and Supplementary Table 1). Post-embryonic PIN2 mRNA is strongly reduced in triple mutant roots before differentiation (Fig. 1k, 1). Therefore, PLT1, PLT2 and PLT3 redundantly control expression of multiple PIN genes in the embryonic and postembryonic root.

$b b m-1$ and $b b m-2$ mutant alleles carry T-DNA insertions before and in the beginning of the first AP2 domain, respectively (Supplementary Fig. 2). Truncated transcripts are detected by RT-PCR and may be translated, but genetic interactions (described below) suggest that the insertions cause loss-of-function effects. plt $3^{-1-} \mathrm{bbm} \mathrm{m}^{-/-}$ double mutants have a shorter root and root meristem than either single mutant (Fig. 1f, and Supplementary Fig. 3).

Intriguingly, the progeny of plants segregating different plt and $\mathrm{bbm}$ allele combinations lack root and hypocotyl (Fig. 1e, lower inset) at significant frequencies (Supplementary Table 2), reaching $\sim 10 \%$ of the progeny of selfed plt $1^{-1-} p l t 2^{+/-} p l t 3^{-/-} b b m-2^{-/-}$. These defects initiate in the early basal embryo (Supplementary Fig. 5) and resemble those in mutants of the auxin response factor MONOPTEROS ${ }^{12}$ and the auxin perception machinery ${ }^{13,14}$. PLT genes do not seem to strongly perturb early global auxin-dependent patterning processes, as suggested by essentially normal cotyledon vasculature in the triple mutant (Supplementary Fig. 4). Segregation 
of plt 2 in a homozygous $b b m$ background and vice versa yields $\sim 25 \%$ early arrested embryos, and homozygous double mutants could not be recovered, indicating a redundant function in early embryogenesis (data not shown).

Ectopic root structures are initiated by constitutive embryonic expression of PLT genes ${ }^{3}$ and after induction of BBM expression ${ }^{15}$. To test whether PLT induction induces a developmental switch to root development, we expressed a PLT2-GR fusion protein that complements $p l t 1^{-1-}$ plt $2^{-1-}$ after dexamethasone (dex) induction, when driven by its own promoter (Supplementary Fig. 6). When $35 S-P L T 2-G R$ is activated by application of dex, roots are produced from the shoot apex (Fig. 1m, n). Our gain- and loss-of-function experiments indicate that PLT genes are master switches for root development.
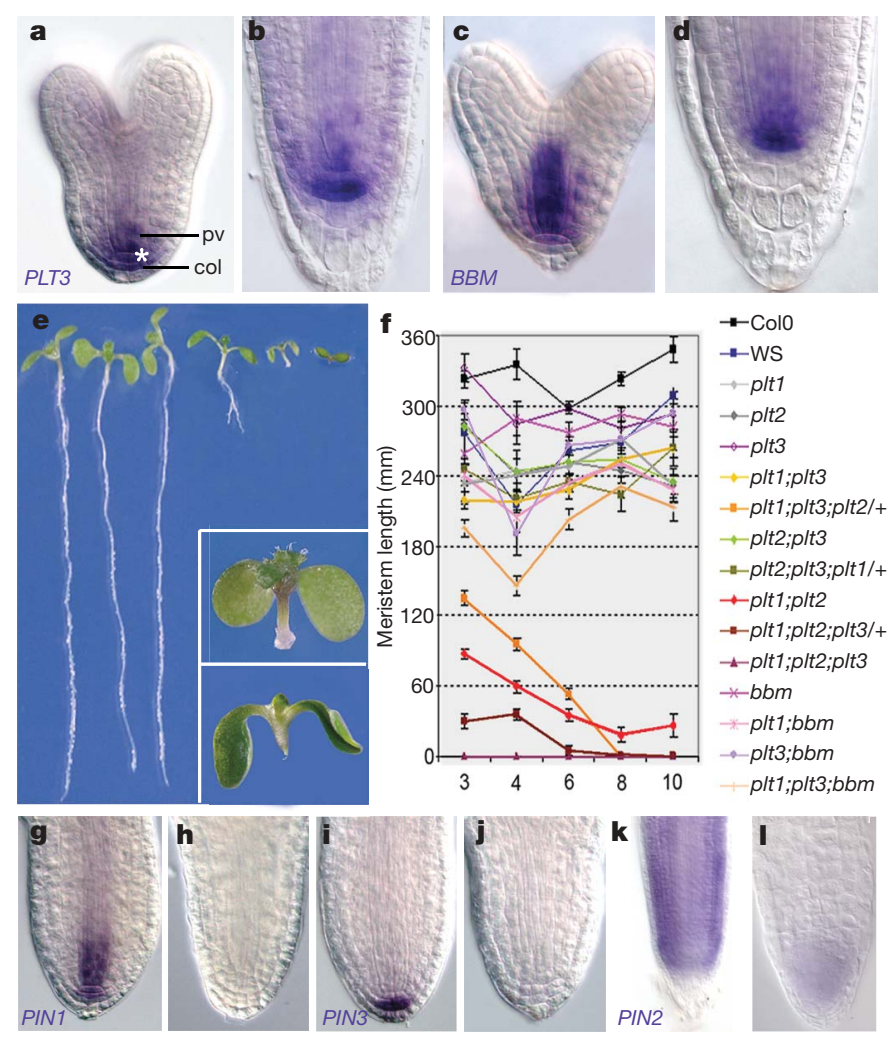

\section{- Col0}

- WS

$\rightarrow$ plt1

$\rightarrow-p / t 2$

$\rightarrow p / t 3$

plt1;plt3

-plt1;plt3;plt2/+

+ plt2;p/t3

- plt2;p/t3;plt1/+

$\rightarrow$ plt1;plt2

- - plt1;plt2;plt3/+

$₫-p / t 1 ; p / t 2 ; p / t 3$

$-x-b b m$

- plt1;bbm

- plt3;bbm
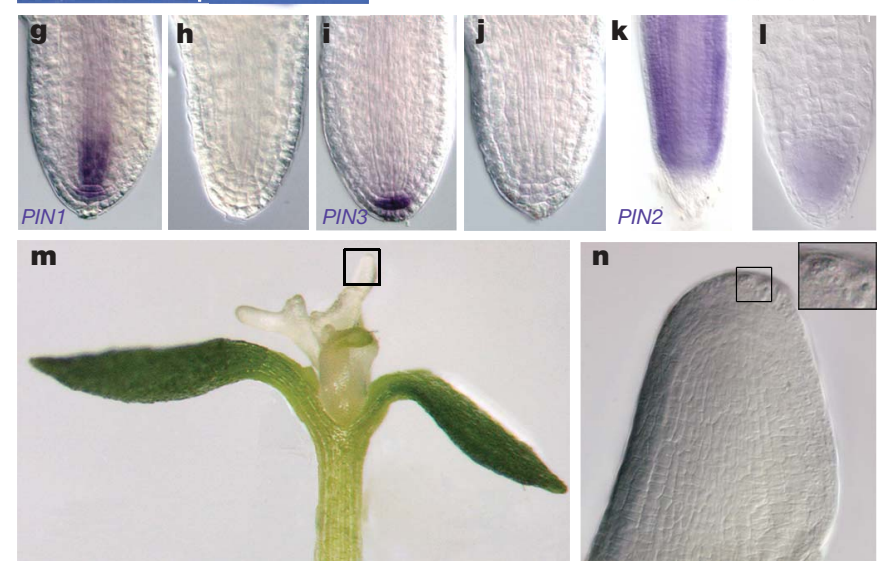

Figure 1 | Four PLT genes promote root formation. a-d, In situ

hybridization with PLT3- (a, b) and BBM- (c, d) specific probes in wild-type embryos at heart-stage (a, c), and in roots of 3 d.p.g. wild-type plants (b, d). Asterisk, quiescent centre; pv, provasculature; col, columella. e, Seedlings 10 d.p.g., from left to right: wild type, plt $3^{-/-}, b b m-1^{-1}$ plt $1^{-1-}$ plt $2^{-1-}$, plt $1^{-1-}$ plt $2^{-1-}$ plt $3^{-/-}$and a plt $1^{-/-}$plt $2^{+/-}$plt $3^{-1-}$ bbm$1^{-/-}$segregant. Insets show magnification of plt $1^{-I-}$ plt $2^{-/-}$plt $3^{-/-}$mutant (upper) and $p l t 1^{-/-} p l t 2^{+/-} b b m-1^{-/-}$segregant (lower). f, Meristem size in wild type (Col0 and WS) and plt mutants at the indicated d.p.g. For each data point, $n=10$ to 50; error bars, s.e.m. g-I, In situ hybridization using PIN probes on wild-type $(\mathrm{g}, \mathbf{i})$ and plt $1^{-1-}$ plt $2^{-1-}$ plt $3^{-l-}(\mathbf{h}, \mathbf{j})$ torpedo-stage embryos and wild-type (k) and plt ${ }^{-1-}$ plt $2^{-1-}$ plt $3^{-1-}$ mutant (I) 2 d.p.g. seedlings. m, Shoot of 9 d.p.g. 35S-PLT2-GR plant 6 days after dexamethasone application. $\mathbf{n}$, Magnification reveals cellular organization of ectopic root including columella starch granules. plt1 $1^{-l-}$ plt $2^{+/-}$plt3 $3^{-/-}$mutants have intermediate root and meristem size between $p l t 1^{-/-}$plt $3^{-/-}$and $p l t 1^{-1-} p l t 2^{-1-} p l t 3^{-1-}$ (Fig. le, f, and Supplementary Fig. 3) and $50 \%$ of plt $1^{-1-}$ plt $2^{+/-}$ plt $3^{-1-} \mathrm{bbm}^{-1-}$ seedlings have shorter roots than $\mathrm{plt}^{-/-} \mathrm{plt3}^{-/-}$ $\mathrm{bbm}^{-1-}$, whereas $50 \%$ have no primary root (Supplementary Table 2). plt3 alleles are also semi-dominant, because growth and meristem maintenance defects in $p l t 1^{-/-}$plt $2^{-/-}$plt $3^{+/-}$seedlings are intermediate between $p l t 1^{-l-}$ plt $2^{-/-}$and $p l t 1^{-l-}$ plt $2^{-l-}$ plt $3^{-/-}$(Fig. 1f, and Supplementary Fig. 3). The semi-dominance of plt2 and plt3 loss-of-function alleles indicates dose-dependent activity.

To test whether PLT genes equally contribute to PLT 'dosage', we transformed $p l t 1^{-1-}$ plt $2^{-1-}$ double mutants with PLT1, PLT2, PLT3 and $B B M$ genes fused to the yellow fluorescent protein gene YFP and driven by the full PLT2 promoter. In independent lines with similar overall YFP levels, PLT1 and PLT2 fully complemented and PLT3 and $B B M$ partially complemented root growth in the double mutant. All PLT proteins rescued columella stem cell activity (Supplementary Figs 6 and 7). Thus, total PLT levels and to some extent intrinsic differences in PLT protein activity contribute to root growth and stem cell maintenance.

Transgenic lines carrying complete promoters of the PLT genes fused to the cyan fluorescent protein gene CFP reveal highest promoter activity in the stem cell niche, consistent with mRNA levels, but they also show graded activity in the proximal meristem (Fig. 2a-d). Gradients can be observed in epidermal surface views, excluding quenching effects, and they are specific to PLT promoters (Fig. 2e). To analyse whether this promoter activity drives a PLT protein gradient, we combined the PLT-YFP fusions with their corresponding full promoters. PLT1 and PLT2 gene fusions complemented plt $1^{-/-}$plt $2^{-/-}$mutants (Supplementary Figs 6 and 7, and data not shown).
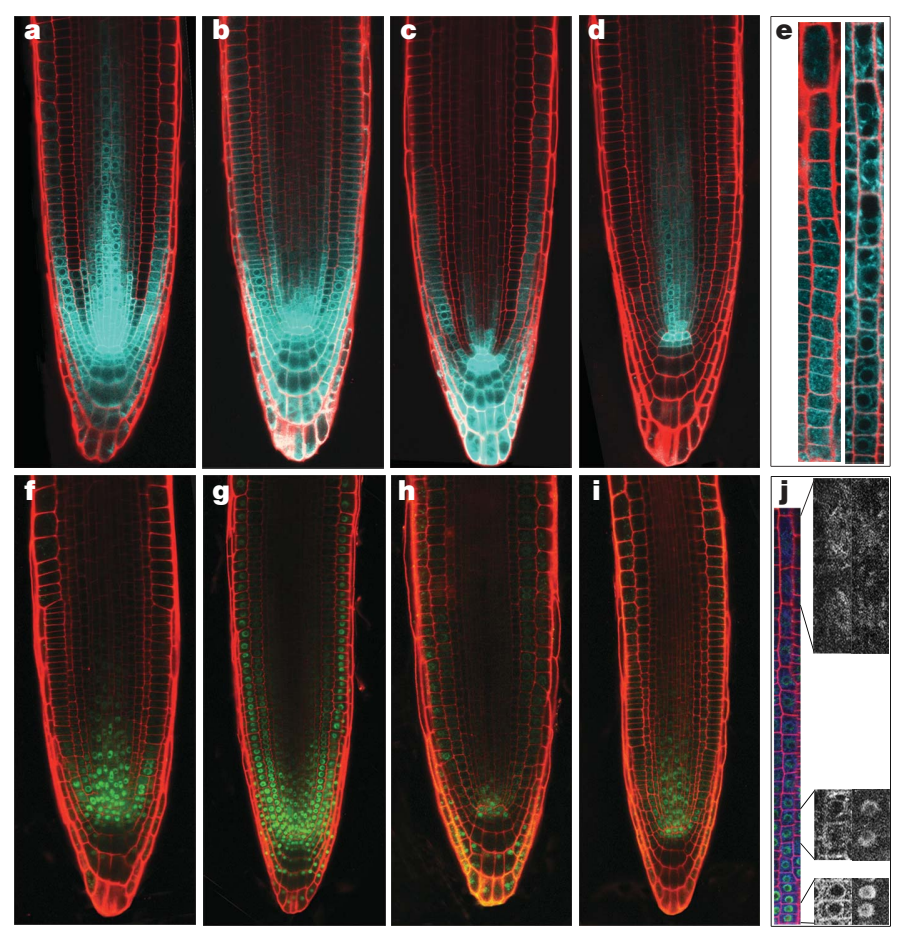

Figure 2 | PLT promoter activity and PLT protein fusions display gradients. a-d, CFP reporter driven by full-size promoters of PLT1 (a) PLT2 (b) PLT3 (c) and BBM (d). e, Epidermal gradient of PLT2 (left) but not $\mathrm{RCH} 2$ (right) promoter. $\mathbf{f}-\mathbf{i}$, YFP reporter fused in-frame to genomic fragments of PLT1 (f), PLT2 (g), PLT3 (h) and BBM (i). j, Co-localization in one plant of $P L T 2$ transcriptional (CFP, left magnification) and translational (YFP, right magnification) fusion viewed in different regions using separate channels. 
All PLT protein fusions revealed conspicuous gradients that extend into the transit-amplifying cells and, for the PLT2 and PLT3 fusions, into the elongation zone (Fig. 2f-i). The promoter and protein gradients fully match when combined in one plant (Fig. 2j). We previously reported accumulation of PLT transcripts in the stem cell area $^{3}$, but, after extended staining, PLT1 in situ hybridizations also reveal a broader expression domain (Supplementary Fig. 8). We concluded that PLT promoter activity leads to protein gradients with maximum expression in the stem cell niche. PLT1 and PLT2 expression maxima broadly encompass the niche, whereas PLT3 and BBM are more restricted.

We asked whether differences in PLT expression domains affect the ability of PLT proteins to compensate for redundant partners. Indeed, PLT1 and PLT2 only partially complement a plt $1^{-/-} p l t 2^{-1-}$ mutant when driven by the BBM promoter (Supplementary Figs 6 and 7).

Our experiments suggested that the PLT protein concentration gradient instructs different outputs in different regions, even though each gene slightly differs in activity and expression profile. We therefore tested whether altering the level or shape of the PLT2 gradient affects the position of developmental boundaries. We expressed the PLT2-YFP fusion in plt $1^{-1-}$ plt $2^{-1-}$ mutants under the $\mathrm{RCH} 2$ promoter, which has low activity in the stem cell area but is active in meristematic and elongating cells at a level comparable to that of the PLT2 promoter (Fig. 3d-g). RCH2-PLT2-YFP prolongs transitamplifying cell divisions but fails to maintain stem cells at 7 d.p.g. (Fig. 3b, d). The transit-amplifying cell pool is lost at $12 \mathrm{~d} . \mathrm{p} . \mathrm{g}$. (Fig. 3c). We concluded that intermediate PLT levels in the meristem promote transient cell cycling.

To validate that meristem size is controlled by a PLT gradient, we analysed $p l t 1^{-1-} p l t 2^{-1-}$ mutants complemented with the PLT2-YFP construct driven by a truncated $1.3-\mathrm{kb}$ PLT2 promoter fragment ( $p$ PLT2s). This truncated promoter drives significant expression in the stem cell area but the gradient declines more rapidly (Fig. 3e, f). Accordingly, stem cells are rescued but root and meristem sizes are $\sim 50 \%$ smaller (Supplementary Fig. 7). The amount of YFP signal per mid-nuclear section in the stem cell zone, halfway the meristem, and in the first expanding cells, provides three clearly separated intensity ranges that match with zonation in the full- and truncated-promoter driven gradients (Fig. 3g), suggesting that the PLT2 gradient defines meristem zonation.

A dose-dependent gradient model predicts that PLT overexpression shifts the meristem boundary. Indeed, dex induction of 35S-PLT2-GR plants promotes continuous growth of the transitamplifying cell pool and meristem size increases (Fig. $4 \mathrm{a}-\mathrm{c}$ ). Ink toner marks marking the elongation zone boundaries at the time of induction reveal that PLT overexpression sustains cell division only in cells that are still cycling and inhibits cell expansion in the elongation zone. These data reinforce the idea that distinct PLT levels dictate cell proliferation and mitotic exit.

The auxin response marker DR5-GUS ${ }^{8}$ and PIN3 transcription do not change in 35S-PLT2-GR plants just before the onset of meristem size expansion, but only at later stages, indicating that PLT-induced expansion of the division zone is not caused by rapid changes in PIN expression (Supplementary Fig. 9).

Notably, the stem cell area in PLT2-GR plants is not altered after induction (Fig. 4c). The RETINOBLASTOMA (RBR) pathway was recently identified as an independent stem cell input ${ }^{16}$, so we reasoned that this pathway might still limit stem cell pool size in the presence of higher PLT levels. Therefore, we combined a root-specific RNA interference (RNAi)-mediated silencing construct ( $R C H 1$ $R B R i)^{16}$ with $35 S-P L T 2-G R$. After induction with dex in the double transgenic, root meristem size increases as in 35S-PLT2-GR, but clusters of dividing cells in the root cap area expand beyond that seen in RCH1-RBRi alone (Fig. 4d, e). Moreover, periclinal divisions normally associated with stem cells occur throughout the proximal area (Fig. 4f). These data suggested that the high expression region of the PLT gradient can be instructive for stem cell fate. Dramatic support for this notion is provided by duplications of the distal stem cell area in $\sim 10 \%$ of $R C H 1-R B R i ; 35 S-P L T 2-G R$ root meristem zones (Fig. 4g-i). We concluded that high PLT levels define the stem cell domain, confirming PLT dosage-dependent stem cell specification.
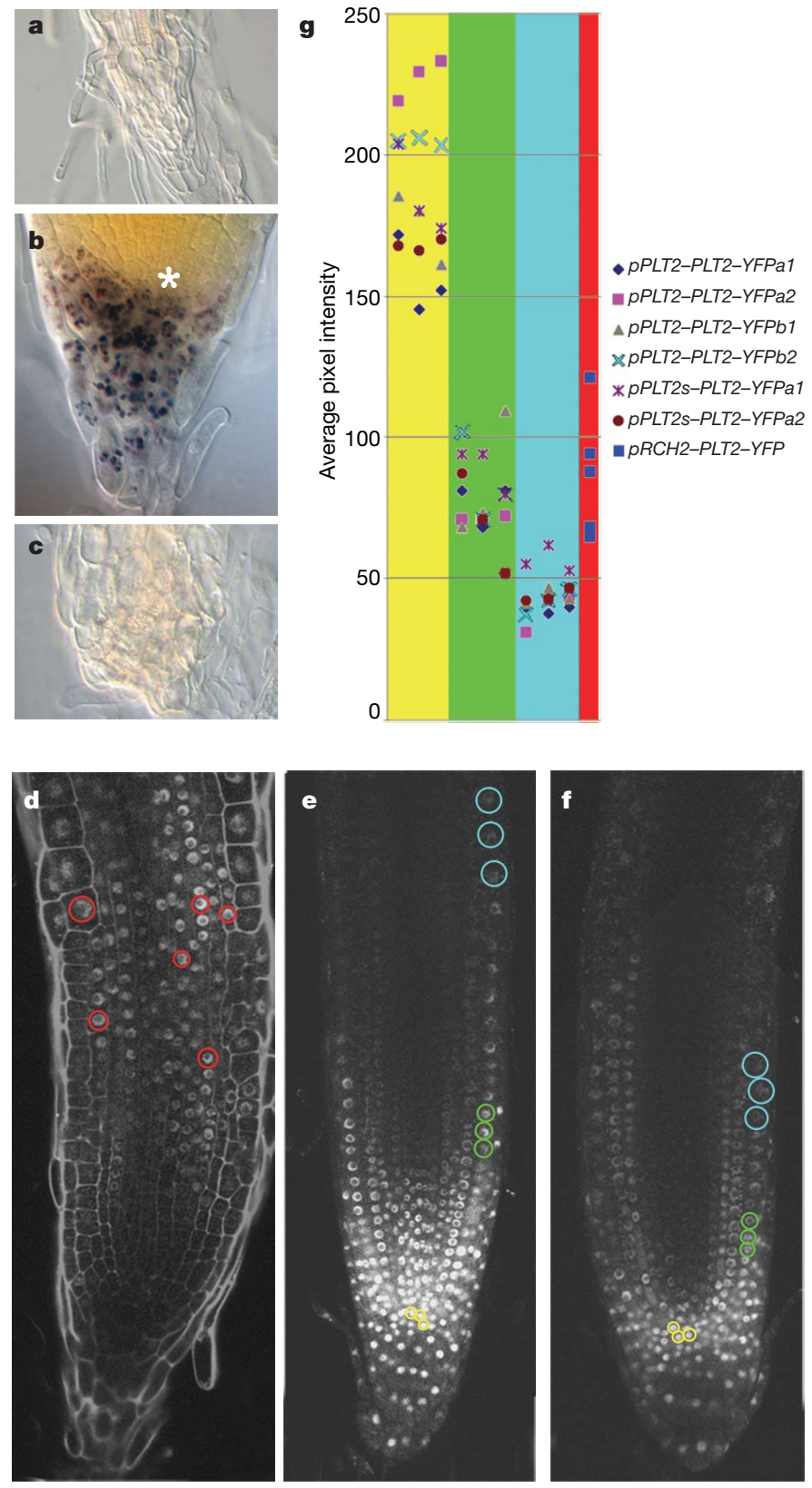

Figure 3 | PLT expression regulates stem cell maintenance and meristem boundary. a-d, Meristem prolongation but not stem cell rescue in $\mathrm{RCH} 2$ PLT2-YFP plants. Nomarski optics image of root tip of 7 d.p.g. plt1;plt2 (a), and of plt1;plt2 RCH2-PLT2-YFP at 7 d.p.g. (b) and 12 d.p.g. (c). Starch granule staining (brown) shows no rescue of columella stem cells below the quiescent centre. Confocal view of 7 d.p.g. plt1;plt2 RCH2-PLT2-YFP root (d) shows that the meristem is rescued and reveals no expression of PLT2-YFP in the stem cell area. Asterisk in b, quiescent centre. e, $\mathbf{f}$, Promoter truncation shifts the meristem boundary. CLSM views at identical pinhole and laser settings for RCH2-PLT2-YFP (d), pPLT2-PLT2-YFP (e) and $p P L T 2 s-P L T 2-Y F P(\mathbf{f})$. $\mathbf{g}$, Quantification of fluorescence per nucleus in $p R C H 2-P L T 2-Y F P$ transient meristem (red circles in $\mathbf{d}$, and red graph areas), and in stem cells (yellow in $\mathbf{e}, \mathbf{f}$ and graph area), mid-meristem (green in $e, f$ and graph area) and first elongating cells (blue in $\mathbf{e}, \mathbf{f}$ and graph area) of pPLT2-PLT2-YFP and pPLT2s-PLT2-YFP ( $\mathrm{a}$ and $\mathrm{b}$ indicate independent transformants, 1 and 2 indicate different roots). 


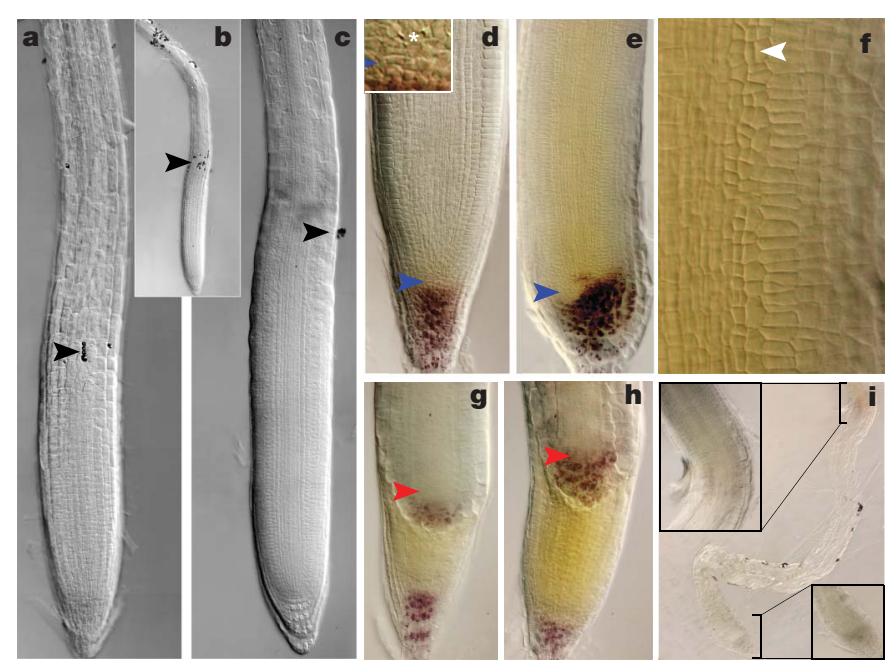

Figure 4 | Inducible expansion of meristem and stem cell area with PLT2-GR fusions. a-c, 35S-PLT2-GR 7 d.p.g. without dex (a) and $1 \mathrm{~d}$ after $5 \mu \mathrm{M}$ dex application $(\mathbf{b}, \mathbf{c})$. Overview shows positioning of ink toner particles that mark the meristem boundary (black arrowhead) and upper elongation zone boundary at the onset of induction (b); the elongation zone boundary is defined as the position where cortical cells rapidly expand. Induced PLT2-GR roots reveal cell division below the meristem boundary and incomplete cell elongation (c). d-f, 35S-PLT2-GR;pRCH1-RBR RNAi plants: 10 d.p.g. without dex revealing the two $R B R i$-induced stem cell layers below the quiescent centre (blue arrowhead, inset), asterisk indicates the quiescent centre (d); with $3 \mathrm{~d}$ of dex application, revealing excessive root cap stem cells (blue arrowhead) and periclinal divisions in the proximal meristem (e); magnification with ectopic periclinal divisions ( $\mathbf{f}$, white arrowhead). $\mathbf{g}-\mathbf{i}$, Duplication of the stem cell area (red arrowheads) and distal cell types (brown starch granules) in $\sim 10 \%$ of 8 d.p.g. 35S-PLT2-GR, $p R C H 1-R B R i$ plants after dex application. Early (g), mid- (h) and late (i) stages of ectopic stem cell centre; note the prolonged activity of both stem cell centres (i, inset).

This effect is normally limited by RBR. Low RBR levels in the RCH1$R B R i$ transgenic display limited expansion of the stem cell domain ${ }^{16}$ because the PLT levels dictated by the gradient are limiting.

Our data indicate that PLT protein gradients define three outputs in the growing root primordium: stem cell programming, mitotic activity and exit to differentiation. Analysis of PLT target genes will be required to assess how much of the response to graded activity is due to additive concentration effects on the same targets and to differences in target specificity.

Although the molecular link between auxin action and PLT gene activation may not be direct $^{3}$, auxin distribution and response systems are essential for correct PLT gene transcription. This raises the possibility that PLT proteins promote stem cells and transitamplifying cells as a graded read-out of auxin distribution. In an accompanying paper, we provide evidence that PIN-mediated polar auxin transport establishes a dynamic gradient spanning the root meristem ${ }^{17}$. Hence it is tempting to speculate that an auxin gradient underlies the observed PLT gradients. Classical morphogen systems were conceptualized as independent from the response system. However, several gradients in animal development involve complicated dynamics (for example, ref. 18) and the static concept of positional information is being challenged ${ }^{19}$. We show that PIN polar auxin transport facilitator expression that is essential for correct auxin distribution is regulated by PLT activity, which is a clear example of entanglement between positional information and its response system.

\section{METHODS SUMMARY}

Plant work. plt1-4 and plt2-2 alleles were described in ref. 3, plt3-1, and bbm-1 and bbm-2 are salk T-DNA insertion lines 127417, 097021 and 067917, respectively, provided by the Signal Insertion Mutant Library (http://signal.salk.edu/).
The T-DNA insertion in PLT3 was confirmed by genotyping. The plt1;plt2;plt3 triple mutant was generated by crossing plt3-1 to plt1-4;plt2-2. bbm-1 and $b b m-2$ were crossed to plt1-4;plt2-2 and plt3-1 and allelic combinations were selected from $\mathrm{F}_{2}$ populations. The T-DNA insertion site on $b b m-1$ and $b b m-2$ lines was verified by genotyping. Primers for genotyping are indicated in Supplementary Table 3. Promoter and genomic sequences were amplified from Col-0 genomic DNA using the primer combinations listed in Supplementary Table 3.. Promoter fragments were fused to the endoplasmic reticulum targeted CFP coding sequence in a pGreenII vector ${ }^{20}$. For translational fusions, PLT genomic sequences were fused at the $3^{\prime}$ end to either the YFP coding sequence or the carboxy-terminal-encoding region of the rat glucocorticoid (GR) receptor ${ }^{21}$ and placed under the control of particular promoters (amplified regions are described in Supplementary Table 3). Promoter swaps were performed by fusing $5.8 \mathrm{~kb}$ of PLT2 and $4.2 \mathrm{~kb}$ of BBM promoter fragments to the YFP-fused PLT genomic sequences. Transgenic plants were generated by transforming Col-0 wild-type or plt1-4;plt2-2 plants, as described ${ }^{22}$.

Phenotype analysis and microscopy. Light microscopy ${ }^{23}$, confocal microscopy and aniline blue staining ${ }^{24}$ of mature embryos was performed as described. Root length was measured, as before ${ }^{3}$. Meristem cell length was measured using ImageJ (v.1.36) and mature cortical cell length as well as fluorescence levels were determined using Zeiss LSM Pascal (3.2SP2) software.

In situ hybridization. Whole-mount RNA in situ hybridization was performed as described ${ }^{11}$. The PLT3 and BBM riboprobes, specific for non-conserved sequences downstream of the AP2 repeats, were prepared from templates amplified from complementary DNA (for primers, see Supplementary Table 3). The PLT1 probe is as in ref. 3; the PIN1, PIN2 and PIN3 probes are as in ref. 25.

\section{Received 5 July; accepted 30 August 2007.}

1. Tabata, T. \& Takei, Y. Morphogens, their identification and regulation. Development 131, 703-712 (2004).

2. Gurdon, J. B. \& Bourillot, P. Y. Morphogen gradient interpretation. Nature 413, 797-803 (2001).

3. Aida, M. et al. The PLETHORA genes mediate patterning of the Arabidopsis root stem cell niche. Cell 119, 109-120 (2004).

4. Skoog, F. \& Miller, C. O. Chemical regulation of growth and organ formation in plant tissues cultured in vitro. Symp. Soc. Exp. Biol. 54, 118-130 (1957)

5. Weigel, D. \& Jurgens, G. Stem cells that make stems. Nature $415,751-754$ (2002).

6. Spradling, A., Drummond-Barbosa, D. \& Kai, T. Stem cells find their niche. Nature 414, 98-104 (2001).

7. $\mathrm{Xu}$, J. et al. A molecular framework for plant regeneration. Science 311, 385-388 (2006).

8. Sabatini, S. et al. An auxin-dependent distal organizer of pattern and polarity in the Arabidopsis root. Cell 99, 463-472 (1999).

9. Nole-Wilson, S., Tranby, T. L. \& Krizek, B. A. AINTEGUMENTA-like (AIL) genes are expressed in young tissues and may specify meristematic or divisioncompetent states. Plant Mol. Biol. 57, 613-628 (2005).

10. Birnbaum, K. et al. A gene expression map of the Arabidopsis root. Science 302, 1956-1960 (2003).

11. Blilou, l. et al. The PIN auxin efflux facilitator network controls growth and patterning in Arabidopsis roots. Nature 433, 39-44 (2005)

12. Hardtke, C. S. \& Berleth, T. The Arabidopsis gene MONOPTEROS encodes a transcription factor mediating embryo axis formation and vascular development EMBO J. 17, 1405-1411 (1998).

13. Hellmann, H. et al. Arabidopsis AXR6 encodes CUL1 implicating SCF E3 ligases in auxin regulation of embryogenesis. EMBO J. 22, 3314-3325 (2003)

14. Dharmasiri, N. et al. Plant development is regulated by a family of auxin receptor $F$ box proteins. Dev. Cell 9, 109-119 (2005).

15. Srinivasan, C. et al. Heterologous expression of the BABY BOOM AP2/ERF transcription factor enhances the regeneration capacity of tobacco (Nicotiana tabacum L.). Planta 225, 341-351 (2007).

16. Wildwater, M. et al. The RETINOBLASTOMA-RELATED gene regulates stem cell maintenance in Arabidopsis roots. Cell 123, 1337-1349 (2005).

17. Grieneisen, V. A., Xu, J., Marée, A. F. M., Hogeweg, P. \& Scheres, B. Auxin transport is sufficient to generate a maximum and gradient guiding root growth. Nature doi:10.1038/nature06215 (this issue).

18. O'Connor, M. B., Umulis, D., Othmer, H. G. \& Blair, S. S. Shaping BMP morphogen gradients in the Drosophila embryo and pupal wing. Development 133, 183-193 (2006).

19. Jaeger, J. \& Reinitz, J. On the dynamic nature of positional information. Bioessays 28, 1102-1111 (2006).

20. Hellens, R. P., Edwards, E. A., Leyland, N. R., Bean, S. \& Mullineaux, P. M. pGreen: a versatile and flexible binary Ti vector for Agrobacterium-mediated plant transformation. Plant Mol. Biol. 42, 819-832 (2000).

21. Aoyama, T. \& Chua, N. H. A glucocorticoid-mediated transcriptional induction system in transgenic plants. Plant J. 11, 605-612 (1997). 
22. Clough, S. J. \& Bent, A. F. Floral dip: a simplified method for Agrobacteriummediated transformation of Arabidopsis thaliana. Plant J. 16, 735-743 (1998).

23. Willemsen, V., Wolkenfelt, H., deVries, G., Weisbeek, P.\& Scheres, B. The HOBBIT gene is required for formation of the root meristem in the Arabidopsis embryo. Development 125, 521-531 (1998).

24. Bougourd, S., Marrison, J. \& Haseloff, J. Technical advance: an aniline blue staining procedure for confocal microscopy and 3D imaging of normal and perturbed cellular phenotypes in mature Arabidopsis embryos. Plant J. 24, 543-550 (2000).

25. Friml, J. et al. AtPIN4 mediates sink-driven auxin gradients and root patterning in Arabidopsis. Cell 108, 661-673 (2002).
Supplementary Information is linked to the online version of the paper at www.nature.com/nature.

Acknowledgements We thank the Netherlands Genomics Initiative (M.L.) and the Portuguese Foundation for Science and Technology (C.G.) for funding, A. Shimotohno and J. M. Perez-Perez for sharing data and Frits Kindt for photography.

Author Information Reprints and permissions information is available at wWw.nature.com/reprints. Correspondence and requests for materials should be addressed to B.S. (b.scheres@uu.nl). 